\title{
Facilitação de Comércio e Impactos sobre o Comércio Bilateral $^{+}$
}

\author{
- Maurício Jorge Pinto de Souza* - Heloísa Lee Burnquist**
}

\begin{abstract}
Resumo
Este trabalho avalia os efeitos da facilitação de comércio, definida em termos da simplificação dos procedimentos de fronteira, sobre o padrão de comércio bilateral de um conjunto de 43 países que inclui o Brasil e alguns de seus principais parceiros comerciais. Para isso são construídos, para cada país, dois índices aplicados à simplificação de procedimentos de fronteira: um índice de simplificação de procedimentos de exportação e outro para a simplificação de procedimentos de importação. Um modelo gravitacional é utilizado para estimar a relação desses índices com o padrão de comércio entre os países. Os resultados sugerem que melhorias nas áreas de facilitação de comércio, que levariam a um incremento relativo dos índices de simplificação, podem estimular os fluxos de comércio entre os países.
\end{abstract}

\section{Palavras-chave}

Facilitação de comércio, simplificação, modelo gravitacional.

\begin{abstract}
This work evaluates the effects of trade facilitation, defined as the simplification of border procedures, upon bilateral trade for a set of 43 countries, including Brazil and some of the country's major trade partners. For that purpose, two indexes related to simplification of border procedures were constructed for the selected countries: an index about simplification of export procedures and other about simplification of import procedures. A gravity model is used to estimate the relationship between these indexes and the trade pattern between the countries. The results suggest that improvements in trade facilitation lead to higher indexes of trade simplification indexes which may stimulate bilateral trade within the countries.
\end{abstract}

\section{Keywords}

Trade Facilitation, simplification, gravity model.

\section{JEL Classification}

F13

$+\quad$ Os autores agradecem ao suporte financeiro do CNPq.

* Professor Doutor da Faculdade de Economia, Administração e Contabilidade de Ribeirão PretoFEARP E-mail: mpjsouza@fearp.usp.br - Endereço para contato: Av. dos Bandeirantes, 3900 Monte Alegre - Ribeirão Preto - São Paulo - CEP: 14040-905.

** Professora Associada da Universidade de São Paulo, Escola Superior de Agricultura Luiz de Queiroz (ESALQ/USP) - Departamento de Economia, Admnistração e Sociologia - DEAS - Ph.D. Cornell University - E-mail: hlburnqu@esalq.usp.br - Endereço para contato: Av. Pádua Dias, 11

Piracicaba - São Paulo - CEP: 13400-970.

(Recebido em maio de 2010. Aceito em setembro de 2010). 


\section{Introdução}

A facilitação de comércio pode ser definida como um conjunto de políticas que reduzem os custos para exportar e importar (HELBLE; SHEPHERD; WILSON, 2007; SADIKOV, 2007; SHEPHERD; WILSON, 2008). Essa definição incorpora os chamados "elementos de fronteira" (border elements), tais como a eficiência dos portos, a administração alfandegária e também os elementos denominados "dentro da fronteira" (inside the border), por exemplo, o ambiente regulatório.

Atualmente duas dinâmicas no sistema de comércio internacional que sugerem que a facilitação de comércio é particularmente importante para o crescimento do intercâmbio comercial entre os países. De um lado, a redução das tarifas e das outras formas "tradicionais" de proteção têm aumentado a importância relativa dos procedimentos de fronteira como determinantes dos custos de transação envolvidos no comércio internacional (OECD, 2003). Os prejuízos enfrentados pelas empresas em função dos atrasos portuários, da ausência de transparência e previsibilidade, da burocracia e procedimentos aduaneiros complicados e desatualizados são, em muitos casos, maiores que os custos das tarifas (OECD, 2005).

A segunda dinâmica está relacionada ao processo de reforma comercial. O colapso das negociações da Rodada Doha na Organização Mundial do Comércio - OMC implica que aqueles países que esperavam avanços na direção da reforma comercial terão de buscar alternativas. Nesse sentido, a facilitação de comércio desponta como um caminho alternativo para os países ampliarem o comércio exterior (SHEPHERD; WILSON, 2008).

A importância dos custos de comercialização associados ao comércio internacional contribuiu para que estudos e análises empíricas quanto aos efeitos da facilitação de comércio ganhassem importância para a disciplina da economia e do comércio internacional nos últimos anos. Entretanto, a literatura empírica sobre facilitação de comércio ainda é relativamente limitada. As pesquisas empíricas relacionadas a esse tema têm-se deparado com três desafios: definir e mensurar a facilitação de comércio; escolher a abordagem metodológica para estimar a importância da facilitação para os fluxos comerciais; e delinear cenários para simular os efeitos da facilitação de comércio sobre o intercâmbio comercial (WILSON; MANN; OTSUKI, 2004).

O presente trabalho traz contribuições para as discussões desses dois primeiros tópicos, avaliando os efeitos da facilitação de comércio, definida em termos da simplificação dos procedimentos de fronteira sobre o padrão de comércio bilateral 
de um conjunto de 43 países que inclui o Brasil e alguns de seus principais parceiros comerciais. $^{1}$

A simplificação dos procedimentos de fronteira pode ser entendida como a redução do número de etapas (burocracia) que os exportadores e importadores se defrontam. Procedimentos de fronteira constituídos de diversas etapas e excessivamente burocráticos tendem a ser prejudiciais ao comércio, pois impõem significativos custos de informação para importadores e exportadores potenciais (HELBLE; SHEPHERD; WILSON, 2007). Além disso, avaliar a facilitação de comércio sob a ótica da simplificação dos procedimentos de fronteira é um dos assuntos que norteiam as discussões atuais sobre a facilitação de comércio na OMC.

Este artigo está estruturado em cinco seções, sendo esta introdução a primeira delas. A seção 2 apresenta uma revisão bibliográfica sobre as evidências empíricas relacionadas à facilitação de comércio; na sequência, a seção 3 abrange a metodologia utilizada no presente estudo: os indicadores de simplificação dos procedimentos de fronteira e o modelo gravitacional; a seção 4 contém os resultados e, por fim, são apresentadas algumas conclusões.

\section{Evidências Empíricas dos Impactos da Facilitação de Comércio}

Diferentes abordagens e metodologias têm sido empregadas para avaliar os efeitos da facilitação, tendo predominado, contudo, o emprego dos modelos de equilíbrio geral computável e do modelo gravitacional (DUVAL, 2006). Além disso, grande parte dos estudos existentes abrange as economias da APEC, dado o compromisso público assumido pelos países que compõem esse bloco comercial regional. Esse compromisso, com objetivos de facilitação de comércio, tem sido relativamente bem sucedido e é um dos poucos identificados nessa área das negociações internacionais. ${ }^{2}$

Diversos estudos (APEC (1999), Hertel, Walmsley e Itakura (2001), UNCTAD (2001), Fox, Francois and Landoño-Kent (2003), OECD (2003) e Francois, Meijl e Tongerem (2005) estimam os efeitos potenciais da facilitação de comércio sobre o bem-estar, empregando modelos de equilíbrio geral computável. Nesses modelos, a facilitação de comércio é comumente representada como um "progresso técnico" nas atividades de transporte e comercialização. Os aumentos de produtividade são

1 A relação completa dos países incluídos na análise e seu respectivo código de acordo com a ISO são apresentados no ANEXO 1.

2 Wilson, Mann e Otsuki (2003) e Helble, Shepherd e Wilson (2007) apresentam uma ampla discussão sobre as medidas de facilitação de comércio no âmbito da APEC. 
interpretados como medidas de facilitação de comércio que reduzem o tempo gasto com a realização dos procedimentos aduaneiros e alfandegários (OECD, 2003).

Um exemplo é o trabalho da APEC (1999), que utiliza uma versão dinâmica do modelo GTAP (Global Trade Analysis Project) para o ano base de 1996 e com uma agregação de 14 setores e 21 regiões para estimar os impactos da liberalização e de medidas de facilitação de comércio entre os membros da APEC. Os efeitos de medidas de facilitação de comércio são tratados pelos autores como ganhos de produtividade do setor de transporte internacional. Em uma das simulações os autores assumem uma redução nos custos, derivada de medidas de facilitação de comércio, de $1 \%$ para economias industrializadas e países recentemente industrializados (Newly Industrialising Countries - NIEs), e de 2\% para outras economias em desenvolvimento. Os resultados indicam que as medidas de facilitação poderiam ampliar o comércio da APEC em 1,3\% os ganhos para os países membros representam $0,25 \%$ do PIB ou cerca de US $\$ 46$ bi1hões. Nessa mesma linha, os trabalhos de Hertel, Walmsley e Itakura (2001) e UNCTAD (2001) utilizam o modelo GTAP para quantificar os impactos de uma maior harmonização das normas para os negócios eletrônicos (e-business).

Fox, Francois and Landoño-Kent (2003), OECD (2003) e Francois, Meijl e Tongerem (2005) também utilizam a abordagem do "aumento de produtividade", porém inserem na análise um componente de imposto (tax component). Esse componente de imposto é utilizado para representar os custos diretos incorridos pelas empresas com o comércio internacional, tais como os custos incorridos para providenciar a documentação necessária. De maneira geral, os resultados desses trabalhos indicam que facilitação de comércio tem um impacto positivo para o comércio bilateral dos países envolvidos.

As abordagens da facilitação de comércio que utilizam modelos de equilíbrio geral computável dão uma visão total dos possíveis impactos de melhorias nessa área. Porém, conforme destacado por Wilson, Mann e Otsuki (2003, p.6), os trabalhos que avaliam a facilitação de comércio como mudanças nos preços de importação ou nos custos de transporte não fornecem as ferramentas de análise política ou resultados que os tomadores de decisão necessitam. Visando suprir informações dessa natureza, ganha importância a construção de indicadores que captam diferentes aspectos da facilitação de comércio, associados à utilização de modelos gravitacionais como metodologia para avaliar os impactos da facilitação de comércio. Este tipo de análise tem como vantagem indicar os responsáveis pela política como abordar os esforços de facilitação com um maior grau de detalhamento. Nesta área, destacamse os trabalhos de Wilson, Mann e Otsuki (2003, 2004), APEC (2004) e Helble, Shepherd e Wilson (2007). 
O trabalho de Wilson, Mann e Otsuki (2003) analisa o impacto da facilitação de comércio sobre os fluxos comerciais dos países da APEC. Para isso, os autores definem e mensuram a facilitação de comércio usando quatro indicadores. Esses indicadores são construídos a partir de dados que caracterizam a eficiência portuária do país, o ambiente alfandegário, ambiente regulatório e utilização do e-business. A relação entre os fluxos de comércio e os indicadores é estimada com um modelo gravitacional que inclui, além das variáveis tradicionais, tarifas bilaterais aplicadas. A análise enfoca os fluxos bilaterais de produtos manufaturados no período de 1989 a 2000. De acordo com os resultados, melhorias na eficiência portuária levariam a significativos aumentos do comércio. Já os avanços em termos de ambiente alfandegário e do e-business também têm impacto significativo sobre o comércio, porém em menor proporção quando comparado com melhorias nos portos.

Nessa mesma linha Wilson, Mann e Otsuki (2004) estimam um modelo gravitacional com dados de 75 países, com o objetivo de avaliar a relação entre facilitação de comércio e o comércio de produtos manufaturados nos anos de 2001-2002. Os autores definem a facilitação de comércio utilizando indicadores com denominação semelhante, porém composição diferente de Wilson, Mann e Otsuki (2003). Shepherd e Wilson (2008) também avaliaram os impactos da facilitação sobre o comércio bilateral dos países membros da Associação de Nações do Sudeste Asiático (ASEAN), utilizando indicadores semelhantes aos de Wilson, Mann e Otsuki $(2003,2004)$.

Análise conduzida pela APEC (2004) também utiliza a abordagem do modelo gravitacional incluindo na análise quatro indicadores de facilitação de comércio, quais sejam: procedimentos alfandegários (CP), normas e conformidade (SC), mobilidade de negócios $(B M)$ e tecnologia da informação e comunicação (ICT). Os resultados indicam que se ocorrer $10 \%$ de melhoria em cada uma dessas quatro áreas, o crescimento das importações intra-APEC será de $0,5 \%, 0,6 \%, 1,1 \%$ e 1,5\%, respectivamente.

Sadikov (2007) utiliza um modelo gravitacional com dados de 140 países para avaliar os efeitos das barreiras administrativas associadas ao comércio internacional, definidas como o número de documentos exigidos para exportar e o número de procedimentos necessários para o registro de negócios. A primeira é utilizada como variável proxy para os elementos de fronteira, enquanto a segunda avalia as medidas "dentro da fronteira". Os resultados indicam que a cada documento adicional exigido para exportar tem-se uma redução de 4,2\% nas exportações.

Helble, Shepherd e Wilson (2007) avaliaram a facilitação de comércio definida termos da transparência da política comercial no âmbito das economias da APEC. 
A partir de uma série de variáveis que caracterizam a simplificação e a previsibilidade da política comercial, os autores utilizam a técnica estatística de análise fatorial para construir dois índices, denominados: "transparência nas importações" e "transparência nas exportações". A relação entre os fluxos de comércio e esses indicadores é estimada por meio de um modelo gravitacional. Os resultados sugerem que os ganhos de comércio intra-APEC derivados do aumento da transparência podem chegar a US\$148 bilhões, o que significa um crescimento de 7,5\%.

No que se refere à literatura brasileira com relação à facilitação de comércio, não foram encontrados trabalhos nos moldes desses mencionados anteriormente, que destacam os impactos da facilitação de comércio para os fluxos comerciais do Brasil. Assim, diferentemente dos trabalhos existentes na literatura, este trabalho avalia os impactos da facilitação, considerando um grupo heterogêneo de países de diferentes regióes econômicas e que não possuem objetivos acordados de avanços na área de facilitação de comércio. A ideia é destacar a possível relevância da facilitação como determinante do comércio em um cenário em que os países possuem características distintas e um esforço coletivo relativamente menor no que se refere à facilitação de comércio. Os resultados podem ser particularmente importantes para o Brasil, pois permitem avaliar como a facilitação tem atuado como determinando, considerando o ambiente de comércio no qual o Brasil está inserido.

\section{Metodologia}

Este item apresenta uma relação de indicadores empregados para a construção de dois índices aplicados à simplificação de procedimentos de fronteira: um índice de simplificação de procedimentos de exportação e outro para a simplificação de procedimentos de importação. Também a descrição do modelo gravitacional e os procedimentos econométricos adotados para mensurar os impactos da simplificação sobre os fluxos de comércio bilaterais.

\subsection{Indicadores Empregados na Construção dos Índices de Simplificação dos Procedimentos de Fronteira}

Helble, Shepherd e Wilson (2007) desenvolveram indicadores que podem ser utilizados para avaliar a simplificação dos procedimentos de fronteira e outros aspectos da facilitação de comércio. Para o presente trabalho são selecionados três indicadores para compor os índices, quais sejam: 
- Indicador de Desburocratização dos Documentos Exigidos paraImportação/Exportação: Embora a documentação sempre se faça necessária para dar suporte às transações internacionais, tem-se reconhecido que essas exigências impõem custos, nem sempre justificados, aos agentes econômicos envolvidos. Assim, a simplificação de documentos e exigências desnecessariamente dispendiosas traduz-se em menores custos administrativos para exportadores e importadores (HELBLE, SHEPHERD e WILSON, 2007).

- Indicador do Menor Número de Agências de Fronteira: A ideia geral proposta por Helble, Shepherd e Wilson (2007) é de que um número reduzido de agências envolvidas nas operações de comércio exterior implica uma economia de tempo e recursos para as empresas que operam com a administração e com a adequação às exigências.

- Indicador do Menor Tempo de Desembaraço Alfandegário: O tempo de desembaraço alfandegário pode sinalizar a presença de um processo burocrático, complicado e/ou ineficiente. Dessa forma, a redução do tempo de desembaraço alfandegário pode ser entendida como uma simplificação dos procedimentos na fronteira.

Esses indicadores são utilizados para construir índices que mensuram a simplificação dos procedimentos de fronteira nas importações e exportações de um grupo de países. A Tabela 1 apresenta e descreve as variáveis empregadas para avaliar os indicadores mencionados anteriormente e que representam dimensões da simplificação dos procedimentos de fronteira.

As variáveis DocExigImp, NumAgenImp e ClearanceTime são utilizadas para criar um Índice de Simplificação dos Procedimentos de Importação (ISPI). O grupo de variáveis referentes às exportações (DocExigExp, NumAgenExp e ClearanceTime) é empregado na composição de um Índice de Simplificação dos Procedimentos de Exportação (ISPE).

Os índices são construídos tomando-se a média simples das três variáveis padronizadas. ${ }^{3}$ Para a construção das variáveis padronizadas subtrai-se de cada observação o valor médio da variável e posteriormente o resultado é dividido pelo seu desviopadrão. Além disso, de forma a garantir que os valores elevados dos índices possam ser interpretados como maior simplificação, as escalas das variáveis são alteradas após a padronização. ${ }^{4}$

3 A construção dos índices considerando a média simples das variáveis é utilizada também por Wilson, Mann e Otsuki $(2003,2004)$. Uma abordagem alternativa é utilizar a média ponderada para a construção dos índices, como faz Helble, Shepherd e Wilson (2007). Para determinar as ponderações, esses autores utilizam as cargas fatoriais resultantes da análise fatorial. No presente trabalho optou-se por utilizar uma média simples, uma vez que, utilizando a análise fatorial, as cargas fatoriais para cada uma das três variáveis incluídas no ISPI são semelhantes, indicando que as variáveis têm a mesma importância.

4 Por exemplo, aqueles países que possuem ClearanceTime positivo, após a padronização tiveram seu sinal trocado para indicar menor transparência, da mesma forma os países que apre- 


\section{Tabela 1 - Variáveis Relacionadas com os Indicadores de Simplificação: Descrição e Fonte dos Dados}

\begin{tabular}{lcll}
\hline \multicolumn{1}{c}{ Indicador } & Variável & \multicolumn{1}{c}{ Descrição } & Fonte \\
\hline $\begin{array}{l}\text { Desburocratização dos Documentos } \\
\text { Exigidos para Importação/Exportação }\end{array}$ & $\begin{array}{l}\text { DocExigExpi } \\
\text { DocExiglmpi }\end{array}$ & $\begin{array}{l}\text { Número médio de documentos necessários } \\
\text { para importar ou exportar no país i; }\end{array}$ & DoingBusiness (2008) \\
Menor Número de Agências de Fronteira & $\begin{array}{l}\text { NumAgenExpi } \\
\text { NumAgenlmpi }\end{array}$ & $\begin{array}{l}\text { Número médio de agências envolvidas com } \\
\text { exportação ou importação do país i; }\end{array}$ & $\begin{array}{c}\text { Logistics Performance } \\
\text { Index (2007) }\end{array}$
\end{tabular}

Menor Tempo de Desembaraço Alfandegário ClearanceTime i Tempo de desembaraço alfandegário no país i. Logistics Performance Index (2007)

Como as variáveis consideradas na construção dos índices estão padronizadas, o resultado do índice é adimensional, sendo que valores negativos indicam menor simplificação relativa, enquanto valores positivos indicam maior simplificação relativa. Para facilitar a interpretação dos resultados, além de facilitar a análise econométrica, cria-se uma escala para os índices de tal maneira que 0 representa a menor simplificação e 1 representa a maior simplificação. Para realizar essa modificação, o valor mínimo da amostra é subtraído de cada observação e o resultado é dividido pela amplitude. Dessa forma, assegura-se que o país com menor simplificação assume valor zero, enquanto o país com índice mais elevado assume valor $\mathrm{um}^{5}{ }^{5}$

\subsection{O Modelo Gravitacional}

\subsubsection{Modelo Teórico}

Um modelo gravitacional pode ser empregado para mensurar os impactos dos índices de simplificação sobre o padrão dos fluxos de comércio bilaterais. Introduzido por Tinbergen (1962), a intuição do modelo gravitacional advém da teoria gravitacional da física, podendo ser interpretada como uma expressão de que os fluxos bilaterais de comércio são determinados por forças de atração, que correspondem ao tamanho e à renda dos dois parceiros, e por forças de repulsão que se referem à distância geográfica entre os países (CHENG; WALL, 2005, BALDWIN; TAGLIONI, 2006).

O modelo gravitacional tem-se apresentado adequado para modelar os fluxos de comércio entre as economias, constituindo-se em importante ferramenta de tra-

sentaram sinal negativo, após a padronização tiveram o sinal trocado de forma a indicar maior transparência.

5 As mudanças de escala foram também utilizadas por Helble, Shepherd e Wilson (2007) na construção de indicadores de transparência para os países da APEC. 
balho de economistas ligados à economia internacional (HELBLE; SHEPHERD; WILSON, 2007). Diversos autores, dentre os quais Anderson e Van Wincoop (2003, p. 1), têm considerado que a equação gravitacional é um dos modelos de maior sucesso empírico na análise econômica, sendo particularmente adequado para explicar os fluxos de comércio entre dois países.

A despeito da robustez das estimativas dos modelos gravitacionais, o emprego de seus resultados gerou questionamentos devido à ausência de embasamento teórico desta metodologia. Visando preencher a lacuna existente, diversos trabalhos passaram a ser desenvolvidos, sendo que dentre esses trabalhos seminais que propõem uma fundamentação teórica incluem Anderson (1979), Bergstrand (1989) e Deardorff (1995). As discussões teóricas acerca do modelo gravitacional foram reforçadas pelas contribuições posteriores de Anderson e Van Wincoop $(2003,2004)$. Esses autores propõem um embasamento microeconômico fundamentado para a equação gravitacional.

A análise conduzida no presente trabalho adota como referencial teórico o modelo desenvolvido por Anderson e Van Wincoop (2003, 2004), no qual o modelo gravitacional é derivado a partir de uma função utilidade do tipo CES (Constant Elasticity of Substitution), sujeita a uma restrição orçamentária, associada com condições de equilíbrio de mercado. O modelo permite explicar as exportações realizadas entre o país $i$ e o país $j$, referentes a um determinado setor $k$, em um período de tempo $t$, que assume a seguinte forma:

$X_{i j}^{k}=\frac{E_{j}^{k} Y_{i}^{k}}{Y^{k}}\left(\frac{t_{i j}^{k}}{P_{j}^{k} \Pi_{i}^{k}}\right)^{1-\sigma_{k}}$

em que $Y_{i}^{k}$ e $Y^{k}$ são, respectivamente, a produção do país $i$ e a produção agregada (mundial) no setor $k$; $E_{j}^{k}$ é o dispêndio do país $j$ com o grupo de produtos $k$; $\sigma_{k}$ é a elasticidade de substituição entre grupos de produtos; $t_{i j}^{k}$ representa os custos de comércio incorridos pelos exportadores do setor $\mathrm{k}$, no sentido do país $i$ para o país $j$. Os índices $P_{j}^{k} e \Pi_{i}^{k}$ representam índices de preços, sendo também identificados como índices de resistência multilateral ao comércio. Esses índices são introduzidos para indicar que os fluxos de comércio bilateral não dependem somente dos custos de comércio existente entre os dois países, mas também dos custos de comércio com os demais parceiros comerciais. Anderson e Wincoop (2003) mostram que as estimativas do modelo gravitacional podem ser substancialmente melhoradas quando esses fatores são considerados. 
No que se refere aos custos de comercialização, o desenvolvimento teórico de Anderson e Van Wincoop $(2003,2004)$ propõe que $t_{i j}^{k}$ seja uma função log-linear de variáveis observáveis, tais como a distância bilateral e outros fatores relacionados às barreiras comerciais entre as duas economias.

Shepherd e Wilson (2008) destacam que o modelo teórico desenvolvido por Anderson e Van Wincoop $(2003,2004)$ tem sido aceito na literatura relacionada à economia internacional como o modelo padrão.

\subsubsection{Estratégia Empírica}

A abordagem teórica descrita pode ter um tratamento econométrico adequado empregando-se o método de efeitos fixos (EF) (ANDERSON; VAN WINCOOP, 2003; FEENSTRA, 2004; HELBLE; SHEPHERD; WILSON, 2007). Esse método permite controlar a heterogeneidade dos países, efeito de variáveis omitidas não observáveis ou difíceis de mensurar (como os índices de resistência multilateral), de forma a impedir que a sua omissão se expresse na forma de um viés. ${ }^{6}$ (CHENG; WALL, 2005).

Os modelos de efeitos fixos podem ser usados tanto como efeitos fixos por país específico (ou seja, para cada importador e exportador) como efeitos fixos bilaterais (para cada par de países da amostra) (CHENG; WALL, 2005). Dessa forma, considerando que o efeito fixo capta todas as características específicas da economia ou particularidades dos fluxos bilaterais, torna-se necessário identificar a abordagem mais apropriada, considerando o objetivo do trabalho. O presente estudo estima o modelo gravitacional considerando os efeitos fixos bilaterais, dado que o objetivo é captar o impacto de variáveis específicas a cada economia, como, por exemplo, das tarifas de importação e principalmente das variáveis relacionadas à simplificação sobre o fluxo de comércio bilateral. Para captar esses efeitos, criam-se variáveis dummies para os fluxos de comércio entre pares de países, de forma a possibilitar a expressão de diferenças através do intercepto, que varia entre os pares de países. O número de dummies consideradas é de $R(R-1) / 2$, sendo $R$ o número de países da amostra.

6 A heterogeneidade pode se constituir, por exemplo, no motivo pelo qual um dado país exporta diferentes quantidades para dois outros países, mesmo quando os dois mercados importadores têm o mesmo PIB e são equidistantes do primeiro. Isso pode estar relacionado a fatores políticos, históricos, culturais, étnicos, e mesmo geográficos, que afetam o nível de comércio e são correlacionados com as variáveis do modelo. Assim, estimativas que não levam em conta esses fatores podem apresentar um viés de heterogeneidade, indicando que um mesmo volume será exportado para cada um dos dois importadores quando, na realidade, esses volumes não são idênticos. 
O trabalho propõe a utilização de dados de painel desagregados por produtos para estimar o modelo gravitacional. Nesse caso, além das dummies para fluxos de comércio entre pares de países, são adicionadas variáveis binárias para os anos da amostra, introduzidas para controlar um possível viés causado por variáveis omitidas, ou que não são passíveis de mensuração e que variam ao longo do tempo. Adicionalmente, para controlar a dimensão de produtos (capítulos dos Sistema Harmonizado - SH) e do efeito de variação da elasticidade de substituição entre os grupos produtos, incluem-se variáveis binárias para cada Capítulo do SH (96 Capítulos). De acordo com Shephard e Wilson (2008), a utilização de efeitos fixos por pares de países, associada à utilização de variáveis binárias para a dimensão de produto e tempo, proporciona condições aceitáveis com relação à consistência teórica e ao tratamento empírico.

Outro ponto que deve ser destacado na abordagem empírica refere-se à definição dos fluxos comerciais. Apesar de o modelo teórico estar definido em termos das exportações do país $i$ para o país $j\left(X_{i j}^{k}\right)$, grande parte dos trabalhos empíricos utiliza como variável dependente as importações do país $i$ oriundas do país $j\left(M_{i j}^{k}\right)$ Uma das razões para isso é que os dados de importação dos países tendem a ser de melhor qualidade que os dados de exportação (BALDWIN; TAGLIONI, 2006, p.13).

Antes de apresentar o modelo empírico proposto para o presente trabalho, devese definir o termo que expressa os custos de comércio que afetam as exportações do setor $k$ do país $j$ para o país $i\left(t_{i j}^{k}\right)$. Segundo Cheng e Wall (2005), a utilização de efeitos fixos para os pares de países capta os custos de comércio bilaterais não observados diretamente, tais como os custos de transporte, particularidades geográficas e fatores históricos. Assim, procedendo ao controle desses efeitos, definem-se custos de comercialização que interferem no comércio bilateral, como uma função de variáveis específicas a cada economia. Os custos de comércio são delineados de acordo com a equação a seguir:

$\ln \left(t_{i j}^{k}\right)=\beta_{3} \ln \left(1+\tau_{i}^{k}\right)+\beta_{4} \ln \left(b n t_{i}\right)+\beta_{5} \ln \left(I S P I_{i}\right)+\beta_{6} \ln \left(I S P E_{j}\right)$

em que $\left(1+\tau_{i}^{k}\right)$ é a tarifa aplicada ao grupo de produtos $k$ pelo país importador $i$; $b n t_{i}$ é uma medida para a presença de barreiras não tarifárias na economia importadora $i$; $I S P I_{i}$ representa o índice de simplificação dos procedimentos de importação do país $i$ e $I S P E_{j}$ representa o índice de simplificação dos procedimentos de exportação do país $j$. Dessa forma, considerando conjuntamente o modelo teórico apresentado na equação 1 , a estrutura da equação 2 e a definição empírica dos flu- 
xos de comércio bilateral, a especificação econométrica proposta para o presente estudo assume a seguinte forma:

$$
\begin{gathered}
\ln \left(M_{i j, t}^{k}\right)=\alpha_{i j}+\gamma_{t}+\theta_{k}+\beta_{1} \ln \left(Y_{i, t}\right)+\beta_{2} \ln \left(Y_{j, t}\right)+\beta_{3} \ln \left(1+\tau_{i}^{k}\right)+\beta_{4} \ln \left(b_{i n t}\right) \\
+\beta_{5} \ln \left(I S P I_{i}\right)+\beta_{6} \ln \left(I S P E_{j}\right)+\varepsilon_{\mathrm{ij}, \mathrm{t}}^{\mathrm{k}}
\end{gathered}
$$

em que $M_{i j, t}^{k}$ representa as importações realizadas pelo país $i$ do país $j$ no setor $k$ no ano $t ; t=2003$ a 2006, $\alpha_{i j}$ representa os efeitos fixos bilaterais invariantes no tempo; $\gamma_{t}$ representam variáveis binárias para os anos da amostra; $\theta_{k}$ indicam as $k$ variáveis binárias para as categorias de produtos; $Y_{i, t}$ e $Y_{j, t}$ são o Produto Interno Bruto (PIB) do país importador $i$ e exportador $j$ no tempo $t$, respectivamente; e $\varepsilon_{\mathrm{ij}, \mathrm{t}}^{\mathrm{k}}$ é o termo de erro aleatório.

Para verificar a adequação e a robustez do modelo de efeitos fixos estimam-se também o modelo de efeitos aleatórios e o modelo pooled. Para expressar esse último modelo, incluem-se, além das variáveis apresentadas na equação 3 , mais três variáveis $\left(\right.$ dist $_{i j}$, front $_{i j}$ e idioma $\left.{ }_{i j}\right)$, que tradicionalmente aparecem nos modelos gravitacionais que utilizam dados empilhados. ${ }^{7}$

A significância dos coeficientes de efeito fixo é testada empregando o teste F (Chow) (GREENE, 1993). A existência de efeitos individuais é verificada com o teste do Multiplicador de Lagrange de Breusch e Pagan (1980). Caso existam tais efeitos individuais, o modelo estimado por mínimos quadrados envolvendo uma única constante torna-se inadequado. O teste de Hausman é utilizado para verificar se as diferenças nos coeficientes não são sistemáticas, ou seja, se os coeficientes do modelo e os do efeito aleatório são ortogonais entre si. A rejeição da hipótese nula no teste de Hausman indica que a melhor escolha é o modelo de efeitos fixos, tendo em vista que esta condição é essencial no modelo de efeitos aleatórios (GREENE, 1993). Além disso, os dados serão testados para a existência de heterocedasticidade com o teste proposto por Breusch-Pagan. ${ }^{8}$

Além da estimativa do modelo de efeitos fixos por mínimos quadrados ordinários, este trabalho utiliza o método Poisson pseudo-maximum-likelihood (PPML) introduzido por Santos Silva e Tenreyro (2006). Esse método apresenta-se adequado, pois seus resultados são consistentes na presença de heterocedasticidade e de fluxos

7 dist $_{i j}$ é a distância entre os dois países; front ${ }_{i j}$ é uma variável binária que assume valor um, caso os países compartilhem fronteiras comuns e valor zero, caso não compartilhem; $i d i o m a_{i j}$ é representada por uma variável binária igual a um, caso um par de países tenham um mesmo idioma, e zero caso os idiomas sejam diferentes.

8 Maiores detalhes podem ser encontrados em Greene (1993). 
bilaterais que são zero ou missing na base de dados (SANTOS SILVA; TENREYRO, 2006; SHEPHERD; WILSON, 2008). Nesse caso, a equação 3 é expressa na forma multiplicativa seguindo uma função exponencial com a variável dependente expressa em nível, conforme demonstrado por Santos Silva e Tenreyro (2006) e por Siliverstovs e Schumacher (2007). Esse método vem sendo aplicado nos trabalhos recentes que estimam a equação gravitacional, dentre os quais estão Helble Shepherd e Wilson (2007), Liu (2007), Shepherd e Wilson (2008), Anderson e Yotov (2008) e Behrens, Ertur e Koch (2007).

O modelo gravitacional é estimado para o período de 2003 a 2006, considerando dados do Brasil e de outros 42 países que figuram entre os principais parceiros comerciais do país. ${ }^{9}$ Para determinar o tamanho da amostra utilizou-se como critério a disponibilidade de dados para a construção dos indicadores, a importância do país para o comércio internacional mundial e para a balança comercial brasileira. Considerando a base de dados do presente estudo, o comércio entre esses países representou mais de $60 \%$ de todo o comércio internacional no período de 2003 a 2006. Além disso, os países considerados representaram em 2006, 80\% do mercado de destino das exportações brasileiras e foram à origem de $70 \%$ das importações do país.

Os dados de importação, desagregado de acordo com o Sistema Harmonizado (SH) em dois dígitos (SH-2) no período de 2003 a 2006, são obtidos junto ao COMTRADE (Commodity Trade Statistics Data Base). As estatísticas do Produto Interno Bruto (PIB) dos países no período de 2003 a 2006 foram obtidas junto ao Banco Mundial (World Development Indicators). A variável proxy para as barreiras não tarifárias (BNT) de cada país é o equivalente ad valorem médio das BNTs calculadas por Kee, Nicita e Olarreaga (2008). ${ }^{10}$ Já o MACMAP (Market Access $\mathrm{Map}$ ) é a fonte para as informações desagregadas (SH-2) das tarifas aplicadas pelos países. Os dados da distância geográfica entre os países e das variáveis binárias para fronteiras compartilhadas e mesmo idioma são obtidos junto ao banco de dados do Centre D'Estudes Prospectives et d'Informations Internationales, (CEPII, 2008).

9 Os países incluídos na regressão são Argentina, Austrália, Áustria, Bélgica, Brasil, Bulgária, Canadá, Chile, China, Chipre, República Checa, Dinamarca, Estônia, Finlândia, França, Alemanha, Grécia, Hungria, Irlanda, Itália, Japão, Letônia, Lituânia, Luxemburgo, Malta, México, Países Baixos, Nova Zelândia, Paraguai, Polônia, Portugal, Romênia, Rússia, Arábia Saudita, Eslovênia, Eslováquia, África do Sul, Espanha, Suécia, Inglaterra, Estados Unidos, Coréia e Uruguai (ver ANEXO 1).

10 Kee, Nicita e Olarreaga (2008) estimaram o equivalente ad-valorem das barreiras não tarifárias (BNTs) de 78 países. Os autores consideram como componentes das BNTs medidas de controle de preços e de quantidade, medidas de monopólio e regulamentos técnicos. A partir dessas estimativas os autores calculam três diferentes indicadores de restritividade tanto das tarifas quando das barreiras não tarifárias. De acordo com os resultados dos autores, as BNTs contribuem com mais de $70 \%$ da média de proteção mundial. 


\section{Resultados}

\section{1 Índices de Simplificação dos Procedimentos de Fronteira}

Antes de apresentar os Índices de Simplificação dos Procedimentos de Importação e Simplificação dos Procedimentos de Exportação, são apresentadas as informações sobre as variáveis que compõem esses dois índices.

Como já mencionado, uma medida empírica de simplificação dos procedimentos de fronteira é o número de documentos exigidos para a liberação dos bens na fronteira. A desburocratização dos documentos necessários para importar e/ou exportar traduz-se em um comércio mais simplificado, com menores custos administrativos para os agentes envolvidos.

A Figura 1 apresenta a média do número de documentos exigidos para importar e exportar para o Brasil e os parceiros comerciais. Itália, Paraguai e China são os países da amostra onde o maior número de documentos é exigido para importação. Paraguai, Eslováquia e Eslovênia exigem relativamente mais documentos para exportar que os demais países - em média nove documentos. Nota-se também que os países exigem em média mais documentos para importar (7,3 documentos) do que para exportar (5,7 documentos); esse resultado pode estar refletindo um interesse protecionista dos países, em termos de dificultar as importações.

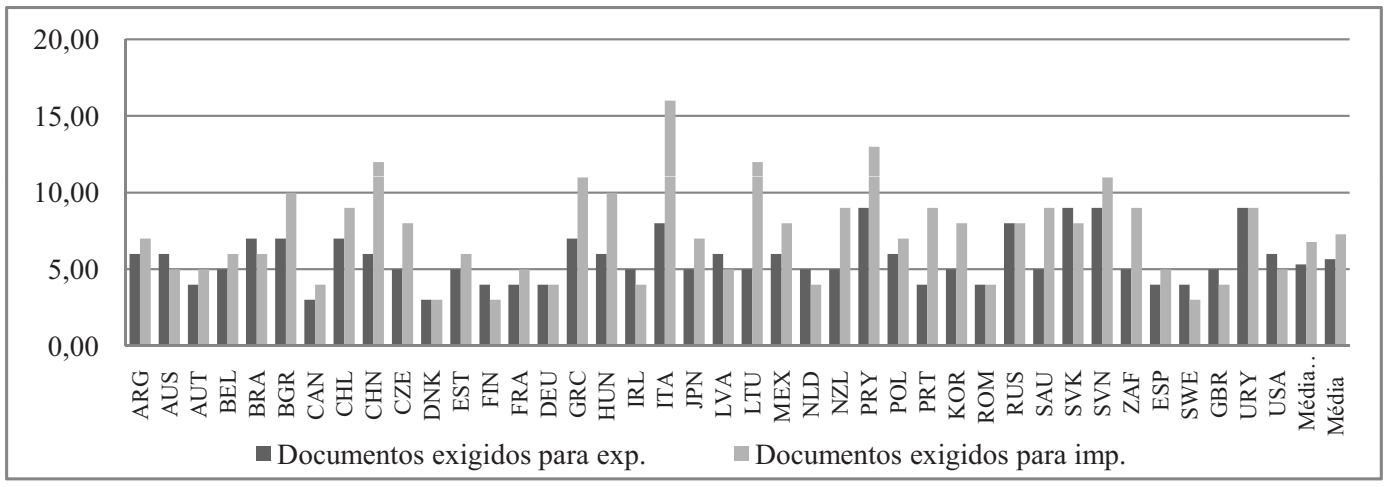

\section{Figura 1 - Número de Documentos Necessários para Importar e Exportar em cada País.}

Fonte: Elaborada a partir de dados do Banco Mundial (2007). ${ }^{11}$

11 Os dados de Doing Business não são disponíveis para três países da União Européia, quais sejam: Chipre, Luxemburgo e Malta de forma que para permitir a construção dos índices e sua posterior inclusão no modelo gravitacional, optou-se por considerar a média do bloco europeu para esses países. 
O relatório Logistics Performance Index (2007) do Banco Mundial contém algumas medidas para elementos de "fronteira" que podem ser utilizadas para avaliar aspectos da simplificação dos procedimentos de fronteira. O tempo de desembaraço alfandegário pode ser entendido como uma medida de simplificação dos procedimentos de fronteira, pois esse tempo pode indicar um processo burocrático, complicado e/ou ineficiente. A primeira coluna da Figura 2 mostra que o Brasil tem o tempo de desembaraço alfandegário mais demorado quando comparado aos demais países da amostra. Esse resultado indica que melhorias nessa área poderiam trazer mais agilidade e menores custos para os exportadores e importadores do país.

Outra medida é o número de agências envolvidas nas operações de comércio exterior, considerando-se que um número reduzido destas agências significa economia de tempo e recursos para as empresas que desejam importar e exportar. A segunda e terceira colunas da Figura 2 apresentam o número médio de agências associadas com a importação e exportação, respectivamente, em cada país da amostra. O interesse protecionista também parece evidente quanto se observa que na média os países possuem maior número de agências associadas à importação $(3,5)$ do que associada a exportação $(3,0)$.

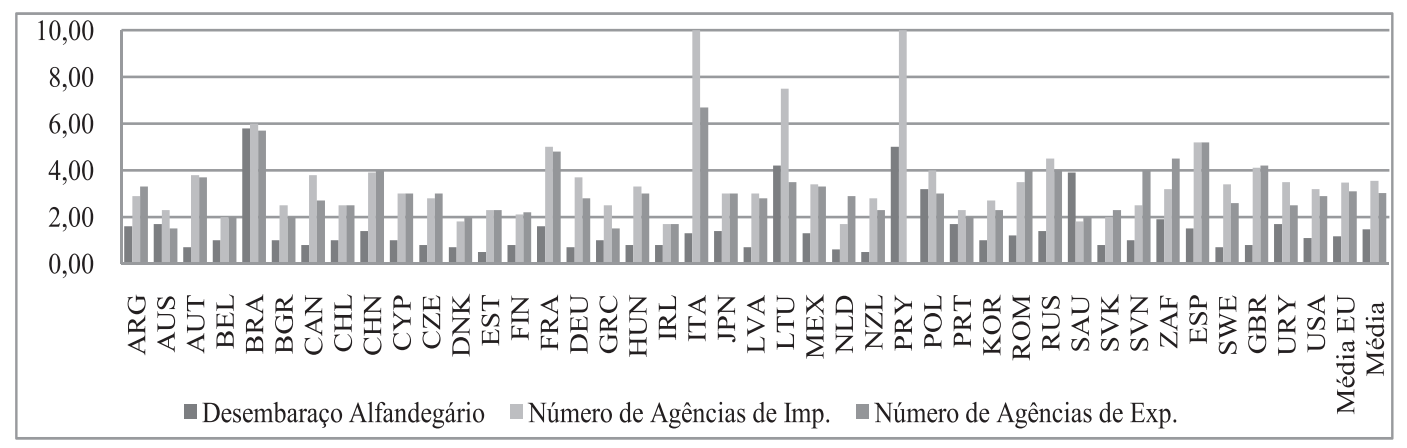

\section{Figura 2 - Tempo de Desembaraço Alfandegário (em dias), Número Médio de Agências Associadas com a Importação e Número Médio de Agências Associadas com Exportação.}

Fonte: Elaborada a partir de dados do Logistcs Performance Index - Banco Mundial (2007).

As informações das Figuras 1 e 2 são sintetizadas em dois índices que mensuram o grau de simplificação dos procedimentos de importação e exportação dos países. A Figura 4 apresenta o Índice de Simplificação dos Procedimentos de Importação ISPI - construído para os países da amostra. O Paraguai aparece no extremo mais 
baixo do índice, seguido de outros três países que apresentam ISPI relativamente abaixo dos demais países da amostra: Itália, Lituânia e Brasil. Enquanto para o Paraguai e para a Itália o principal determinante dessa colocação na classificação geral é o elevado número de agências associadas à importação, para a Lituânia e para o Brasil o problema está associado ao elevado tempo médio de desembaraço alfandegário, que no Brasil é de 5,8 dias.

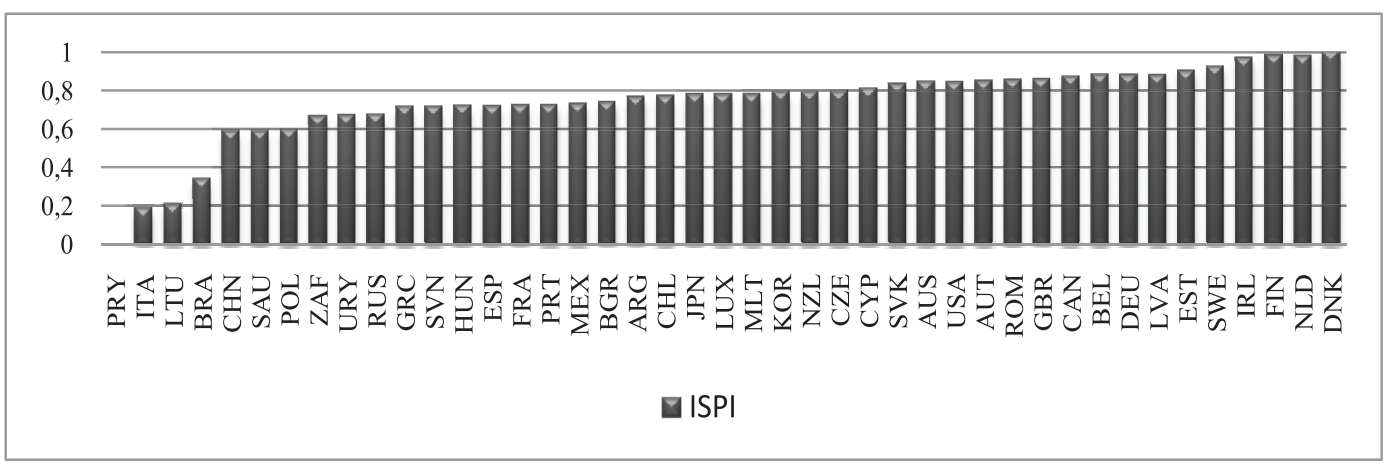

Figura 3 - Índice de Simplificação dos Procedimentos de Importação - ISPI - dos Países Selecionados.

Fonte: Resultados da pesquisa.

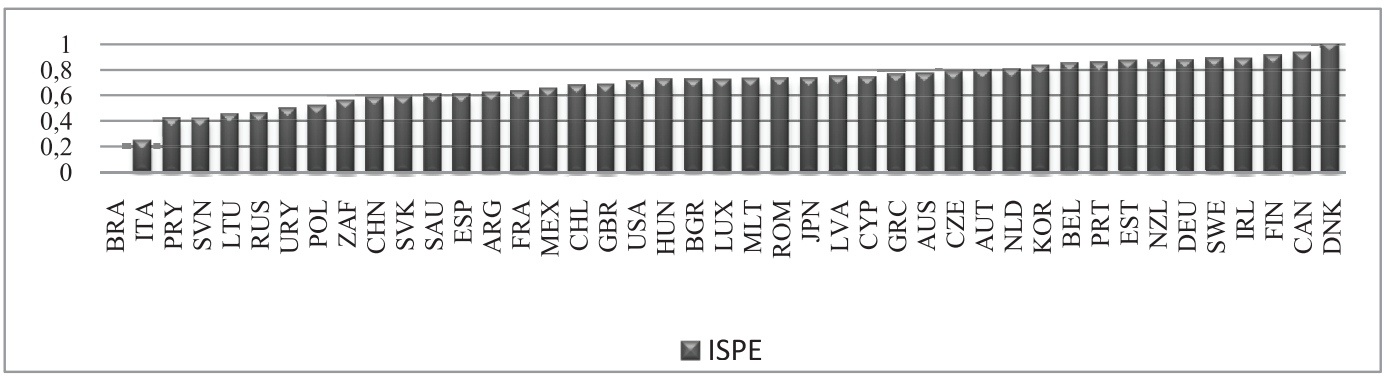

Figura 4 - Índice de Simplificação dos Procedimentos de Exportações - ISPE - dos Países Selecionados.

Fonte: Resultados da pesquisa.

\subsection{Impactos da Facilitação do Comércio no Padrão de Comércio Internacional: Resul- tados do Modelo Gravitacional}

Esta seção contém os resultados das estimativas da equação gravitacional empregada para mensurar os impactos dos índices de simplificação sobre o padrão dos fluxos de comércio bilateral para os 43 países da amostra. 
Como mencionado na metodologia, além das estimativas do modelo gravitacional com efeitos fixos (usando MQO e PPML), estimam-se também o modelo de efeitos aleatórios e o modelo pooled. Entretanto, conforme já discutido, devido à especificação da equação gravitacional, espera-se que as estimativas por MQO do modelo empilhado (Pooled) apresentem viés por não levar em consideração efeitos individuais dos países (BALDWIN; TAGLIONI, 2006, CHENG; WALL, 2005).

Os resultados das estimativas que utilizam dados desagregados em Capítulos do SH são apresentados na Tabela 2. São apresentadas, respectivamente, as estimativas obtidas pelo modelo de regressão Pooled, Efeitos Fixos (MQO e PPML) e Efeitos Aleatórios. O teste proposto por Breusch e Pagan (1979) é empregado no modelo pooled para testar a presença de heterocedasticidade. Como a hipótese nula de variância constante é rejeitada, os erros-padrão foram corrigidos nos quatro modelos estimados, segundo a técnica proposta por White (1980). ${ }^{12}$ Dessa forma, os valores entre parênteses indicam o erro-padrão robusto (robust standard error).

Inicialmente, verifica-se a partir dos resultados do teste $\mathrm{F}$ (Chow), que é aplicado ao modelo de efeitos fixos, e do teste do Multiplicador de Lagrange de Breusch e Pagan (1980), aplicado ao modelo de efeitos aleatórios, que não é possível rejeitar a hipótese de que efeitos de heterogeneidades não observáveis dos países afetam o comércio bilateral. Esses resultados confirmam que os coeficientes obtidos pela regressão pooled apresentam problemas sérios de especificação, sendo tendenciosos e inconsistentes devido à omissão de informações importantes.

Assim, a estimativa do modelo de dados em painel passa a ser a melhor opção a ser avaliada. Os dois métodos de painel mais empregados são o modelo de efeitos fixos (EF) e o modelo de efeitos aleatórios (EA). No primeiro, os termos do intercepto podem variar entre as unidades individuais (ou seja, no caso de pares formados por países importadores e exportadores). O modelo de efeitos aleatórios assume que os interceptos das unidades individuais são aleatoriamente distribuídos e independentes das variáveis explicativas.

O teste de Hausman mostra que a hipótese nula de ausência de correlação entre os efeitos não observáveis de país e as variáveis exógenas do modelo deve ser rejeitada, indicando que o modelo de efeitos fixos deve apresentar estimativas consistentes, apresentando-se, portanto, como o modelo mais eficiente.

Cabe ressaltar que as variáveis binárias para o controle do tempo e da dimensão produto, consideradas nos modelos de efeito fixo (MQO e PPML) e no modelo de

12 Para maiores detalhes, consultar Wooldridge (2002). 
efeitos aleatórios, conforme a equação 3 são estatisticamente significativas para os três modelos.

Tabela 2 - Resultados das Estimativas da Equação Gravitacional Modelo

\begin{tabular}{|c|c|c|c|c|}
\hline & $\begin{array}{c}\text { Regressão } \\
\text { Pooled }\end{array}$ & $\begin{array}{r}\text { Efeitos Fixos } \\
\text { (EF - MQO) }\end{array}$ & $\begin{array}{l}\text { Efeitos Fixos } \\
\text { (EF - PPML) }\end{array}$ & $\begin{array}{c}\text { Efeitos } \\
\text { Aleatórios }\end{array}$ \\
\hline IPib_imp & $\begin{array}{l}0.755^{*} \\
(0.003)\end{array}$ & $\begin{array}{l}0.103^{* *} \\
(0.042)\end{array}$ & $\begin{array}{l}0,675^{*} \\
(0.047)\end{array}$ & $\begin{array}{l}0.703^{*} \\
(0.010)\end{array}$ \\
\hline IPib_exp & $\begin{array}{l}0.853^{*} \\
(0.003)\end{array}$ & $\begin{array}{l}0.284^{*} \\
(0.042)\end{array}$ & $\begin{array}{l}0.631^{*} \\
(0.042)\end{array}$ & $\begin{array}{l}0.884^{*} \\
(0.010)\end{array}$ \\
\hline Itarif & $\begin{array}{l}-1.836^{*} \\
(0.069)\end{array}$ & $\begin{array}{l}-1.450^{*} \\
(0.095)\end{array}$ & $\begin{array}{l}-2,958^{*} \\
(0.783)\end{array}$ & $\begin{array}{l}-1.601^{*} \\
(0.094)\end{array}$ \\
\hline Intb & $\begin{array}{l}-0.114^{*} \\
(0.007)\end{array}$ & $\begin{array}{l}-0.113^{*} \\
(0.008)\end{array}$ & $\begin{array}{l}0.010^{\mathrm{NS}} \\
(0.055)\end{array}$ & $\begin{array}{l}-0.114^{*} \\
(0.008)\end{array}$ \\
\hline Idist & $\begin{array}{l}-0.980^{*} \\
(0.004)\end{array}$ & - & - & - \\
\hline front & $\begin{array}{l}0.780^{*} \\
(0.019)\end{array}$ & - & - & - \\
\hline idioma & $\begin{array}{l}0.645^{*} \\
(0.017)\end{array}$ & - & - & - \\
\hline IISPI & $\begin{array}{l}-0.104^{*} \\
(0.013)\end{array}$ & $\begin{array}{l}0.136^{*} \\
(0.029)\end{array}$ & $\begin{array}{l}0.650^{*} \\
(0.250)\end{array}$ & $\begin{array}{l}0.129^{*} \\
(0.026)\end{array}$ \\
\hline IISPE & $\begin{array}{l}-0.225^{*} \\
(0.015)\end{array}$ & $\begin{array}{l}0.059^{\mathrm{NS}} \\
(0.036)\end{array}$ & $\begin{array}{l}0.788^{*} \\
(0.270)\end{array}$ & $\begin{array}{l}0.045^{\mathrm{NS}} \\
(0.032)\end{array}$ \\
\hline Constante & $\begin{array}{l}21.905^{*} \\
(0.107)\end{array}$ & $\begin{array}{l}-1.002 \\
(2.225)\end{array}$ & - & -33.731 \\
\hline $\mathrm{R} 2$ & 0.3094 & 0.349 & - & 0.348 \\
\hline Observações & 424289 & 424289 & 614976 & 424289 \\
\hline Grupos & & 901 & 901 & 901 \\
\hline Teste F (Chow) & & $235^{*}$ & & \\
\hline Teste Breusch e Pagan & & & & $13000000^{*}$ \\
\hline Teste de Hausman & & & & $1700.34^{*}$ \\
\hline $\begin{array}{l}\text { Variáveis de Controle } \\
\text { Binárias - anos 03-06 }\end{array}$ & Não & Sim & Sim & Sim \\
\hline Binárias - categ. de produtos (96) & Não & Sim & Sim & Sim \\
\hline
\end{tabular}

Nota: Erro-padrão robusto entre parênteses

${ }^{*}$ Denota significância a $1 \%{ }^{* *}$ Denota significância a 5\%. Para os modelos de Efeito Fixo e Efeito Aleatório é reportado o $\mathrm{R}^{2}$ "within groups".

Fonte: Resultados da pesquisa. 
É interessante observar ainda uma forte semelhança entre a significância estatística e os sinais dos coeficientes estimados por efeitos fixos e efeitos aleatórios. Entretanto, os resultados do modelo de efeito fixo estimado pelo método (PPML) são relativamente melhores em termos dos sinais esperados segundo a teoria, bem como em termos da magnitude dos coeficientes estimados. Por exemplo, as elasticidades-renda estimadas pelo modelo EF-PPML são mais próximas à unidade como previsto no modelo teórico da equação gravitacional. O método Poisson pseudomaximum-likelihhod (PPML) apresenta-se adequado, pois seus resultados são consistentes na presença de heterocedasticidade e de fluxos bilaterais que são zero ou missing na base de dados (SANTOS SILVA; TENREYRO, 2006; SHEPHERD; WILSON, 2008). Como a base de dados do presente trabalho é desagregada em capítulos do $\mathrm{SH}$, um número elevado de observações de comércio bilateral são missing, tornando preferível a utilização de um modelo que apresenta resultados robustos na presença desse viés de seleção. Siliverstovs e Schumacher (2007) e Liu (2007) também apresentam argumentos em favor da utilização do método de Poisson pseudo-maximum-likelihhod (PPML) e outras abordagens não lineares. Martinez-Zarzoso, Nowak-Lehmann e Volmer (2007) destacam que o PPML, em função de sua praticidade, pode ser um procedimento padrão para estimar a equação gravitacional. ${ }^{13}$ Sendo assim, são discutidos com maior grau de detalhamento os resultados do modelo de Efeitos Fixos (PPML).

Os coeficientes de Produto Interno Bruto dos países exportadores e importadores apresentam-se positivo e estatisticamente significativo, ao nível de $1 \%$ de significância. Esses resultados são semelhantes aos encontrados na literatura, nos trabalhos de Santos Silva e Tenreyro (2006), Helble, Shepherd e Wilson (2007), Liu (2007) e Siliverstovs e Schumacher (2007). O coeficiente para a tarifa aplicada se apresenta negativo, conforme esperado, e estatisticamente significativo. Esses resultados corroboram os resultados encontrados por Helble, Shepherd e Wilson (2007).

O coeficiente que mede o impacto do equivalente ad valorem (AVE) das barreiras não tarifárias (BNTs) não foi significativo estatisticamente no modelo Efeitos Fixos (PPML). Esse resultado difere do encontrado por Helble, Shepherd e Wilson (2007) e por Disdier, Fontagné e Mimouni (2007), que encontraram coeficientes estatisticamente significativos para o AVE das BNTs. Cabe ressaltar, que diferentemente das estimativas conduzidas por esses autores, o presente trabalho não dispõe de informações desagregadas por capítulos do SH do equivalente ad valorem das barreiras não tarifárias. Dessa forma, a baixa magnitude e significância dos coeficientes estimados podem estar refletindo essa limitação. O impacto marginal das BNTs,

13 No entanto, Martinez-Zarzoso, Nowak-Lehmann e Volmer (2007) demonstram que no caso de previsão fora da amostra os modelos de MQO e de seleção amostral são preferíveis a PPML. 
bem como o significativo impacto das tarifas no comércio, são também verificados quando a estimativa da equação gravitacional é realizada por MQO.

Finalmente, as duas variáveis que representam o foco principal deste trabalho - os índices de simplificação dos procedimentos de importação e o índice de simplificação dos procedimentos de exportações - estão positivamente associadas com o comércio e são estatisticamente significativas. Esse resultado sugere que melhorias nas áreas de facilitação de comércio, que levariam a um incremento relativo dos índices de simplificação, podem estimular os fluxos de comércio entre os países. Cabe ressaltar que tais incrementos relativos demandam reformas políticas e estruturais internas. ${ }^{14}$

Esses resultados corroboram os de Wilson, Mann e Otsuki (2004). Segundo esses autores, a facilitação de comércio através de melhorias nas alfândegas é um caminho possível para reduzir os custos de importação. Adicionalmente, o sinal positivo dos coeficientes para o ISPI e ISPE indica o mesmo resultado dos trabalhos que utilizam modelos aplicados de equilíbrio geral, que modelam a facilitação de comércio como um "progresso técnico" nas atividades de transporte e comercialização. Isso porque esses aumentos de produtividade podem ser entendidos como medidas de facilitação de comércio que reduzem o tempo gasto com a realização dos procedimentos aduaneiros e alfandegários. Nesse contexto, encontram-se os trabalhos de APEC (1999), Hertel, Walmsley e Itakura (2001), UNCTAD (2001), Fox, Francois and Landoño-Kent (2003), OECD (2003) e Francois, Meijl e Tongerem (2005).

Para verificar a robustez dos resultados foram estimados outros dois modelos, considerando sub amostras de produtos. Assim, a Tabela 3 apresenta os resultados das estimativas da equação gravitacional por Efeito Fixo - EF-PPML para diferentes agregações dos capítulos do Sistema Harmonizado. A primeira coluna, equivalente à terceira coluna da Tabela 2, abrange todos os capítulos do Sistema Harmonizado - SH. A segunda coluna exclui matérias-primas (capítulos 1-27) e a terceira coluna exclui manufaturas básicas (capítulos 1-83). Verifica-se que, de maneira geral, os coeficientes estimados apresentaram os sinais esperados e são estatisticamente

14 Cabe ressaltar a possível existência de causalidade reversa entre os indicadores de facilitação e os fluxos de comércio, embora em trabalho com objetivo semelhante, Wilson, Mann, Otsuki (2003) tenham avaliado o problema da endogeneidade nas estimativas do modelo gravitacional para a APEC, obtendo resultados que não indicam um viés significativo. A abordagem adequada na presença de endogeneidade é a utilização de variáveis instrumentais para os índices de facilitação de comércio. Para corrigir esse problema, foram testadas diversas variáveis, tais como variáveis associadas à colonização e à corrupção nos países. No entanto, os resultados indicaram que nenhuma dessas variáveis pode ser considerada um "bom" instrumento para os índices de simplificação. Dessa maneira, os resultados do presente trabalho devem ser avaliados levando em consideração a existência desse possível viés. 
significativos. Além disso, não ocorreram alterações de significância e sinal dos coeficientes quando grupos menores de produtos são considerados.

Tabela 3 - Resultados das Estimativas da Equação Gravitacional pelo Método de Efeitos Fixos (EF-PPML) para Diferentes Agregações dos Capítulos do SH

\begin{tabular}{|c|c|c|c|}
\hline & \multicolumn{3}{|c|}{ Modelo Efeitos Fixos (EF - PPML) } \\
\hline & Todos produtos & $\mathrm{SH}>27$ & $\mathrm{SH}>83$ \\
\hline \multirow[t]{2}{*}{ IPib_imp } & $0,675^{*}$ & $0,665^{*}$ & $0,778^{*}$ \\
\hline & $(0,047)$ & $(0,048)$ & $(0,084)$ \\
\hline \multirow[t]{2}{*}{ IPib_exp } & $0,631^{*}$ & $0,644^{*}$ & $0,880^{*}$ \\
\hline & $(0,042)$ & $(0,044)$ & $(0,085)$ \\
\hline \multirow[t]{2}{*}{ Ltarif } & $-2,958^{*}$ & $-4,842^{*}$ & $-4,791^{*}$ \\
\hline & $(0,783)$ & $(1,239)$ & $(1,333)$ \\
\hline \multirow[t]{2}{*}{ Lntb } & $-0,010^{\mathrm{NS}}$ & $-0,046^{\mathrm{NS}}$ & $-0,101^{\mathrm{NS}}$ \\
\hline & $(0,055)$ & $(0,060)$ & $(0,113)$ \\
\hline \multirow[t]{2}{*}{ IISPI } & $0,650^{*}$ & $0,874^{*}$ & $1,965^{*}$ \\
\hline & $(0,250)$ & $(0,269)$ & $(0,439)$ \\
\hline \multirow[t]{2}{*}{ IISPE } & $0,788^{*}$ & $1,071^{*}$ & $2,455^{*}$ \\
\hline & $(0,270)$ & $(0,874)$ & $(0,512)$ \\
\hline Observações & 614976 & 448420 & 95805 \\
\hline \multicolumn{4}{|l|}{ Variáveis de Controle } \\
\hline Binárias - Anos da Amostra & Sim & Sim & Sim \\
\hline Binárias - Grupos de Produtos & Sim & Sim & Sim \\
\hline Binárias - Pares de Países & Sim & Sim & Sim \\
\hline
\end{tabular}

Nota: Erro-padrão robusto entre parênteses.

*Denota significância a $1 \%$.

Fonte: Resultados da pesquisa.

Os resultados da Tabela 3 indicam que os impactos da facilitação parecem ser mais expressivos para produtos com maior grau de processamento do que para matérias-primas. Produtos com maior grau de processamento podem estar mais sujeitos a problemas associados aos procedimentos de fronteira, relativamente a matérias-primas e produtos agrícolas que são mais homogêneos. Assim os benefícios da facilitação podem depender do tipo de produto em questão, ou ainda do perfil 
da pauta de importação e exportação dos países. Esse tema merece destaque em futuras pesquisas relacionadas à facilitação de comércio.

\section{Considerações Finais}

A intensificação do comércio internacional e a liberalização comercial ocorrida nos últimos anos têm aumentado a importância relativa dos procedimentos de fronteira como determinante dos custos de comercialização associados ao comércio internacional. Nesse contexto, a facilitação de comércio passou a receber destaque no cenário político internacional, tornando-se tópico de discussão na $\mathrm{OMC}$ a partir da Conferência Ministerial de Cingapura de 1996. No entanto, apenas em período recente identifica-se uma intensificação de análises econômicas empíricas para abordar o tema, o que se deve, em grande parte, à dificuldade na obtenção de informações sistemáticas e compatíveis entre os países. O estudo conduzido é importante para destacar a importância do desenvolvimento de informações e estatísticas que possibilitem quantificar a importância de fatores relacionados à facilitação de comércio, seguindo uma linha analítica que se encontra em desenvolvimento também no contexto da literatura internacional.

A análise mostra a importância dessa questão para o Brasil e seus principais parceiros comerciais. Os resultados sugerem que os países têm muito a ganhar com a orientação de políticas que promovam melhorias e contribuam para um melhor posicionamento dos países com relação aos aspectos analisados.

Os resultados indicam que avanços na facilitação de comércio são particularmente importantes para o Brasil. Por exemplo, o país tem o tempo de desembaraço alfandegário mais demorado relativamente aos demais países que compõem a amostra. Aparentemente, o empenho de esforços para essas áreas podem agilizar e reduzir os custos de exportação e importação do país.

De maneira geral, os resultados apresentados indicam que o grau de simplificação dos procedimentos de fronteira dos países, seja como importador ou como exportador, é um importante determinante do padrão de comércio entre os países da amostra. As estimativas sugerem que as barreiras tarifárias constituem-se em importante fator limitante expressivo. No entanto, indica também que os países podem ampliar o comércio bilateral realizando reformas que promovam a facilitação de comércio e reduzam os custos de comercialização internacional. Trata-se de resultado relevante por indicar a importância de ações sobre as quais os países têm maior autonomia na tomada de ações. 
A despeito dos benefícios que as reformas que promovam a facilitação de comércio podem trazer para o comércio bilateral, essas reformas exigem uma série de investimentos como, infraestrutura portuária, investimentos em tecnologia, contratação e formação de capital humano qualificado. Esses investimentos podem ser relativamente elevados para alguns países. Nesse contexto parece interessante que as negociações na área de facilitação de comércio incluam programas de capacity building e assistência para alguns países, principalmente os menos desenvolvidos e em desenvolvimento.

Adicionalmente, as evidências relacionadas aos benefícios da facilitação de comércio sugerem que os ganhos são potencializados quando um maior número de países realiza reformas. Nesse contexto, os resultados sugerem para o Brasil uma postura ativa nas negociações sobre facilitação de comércio, tanto multilateralmente como em negociações com os principais parceiros comerciais, criando, assim, uma alternativa para a ampliação do comércio além da remoção das barreiras tradicionais. Outro ponto a ser destacado com relação ao Brasil é que o tempo de desembaraço alfandegário do país é o mais demorado relativamente dentre os países analisados. Nesse sentido, empenhar esforços nesse campo poderia trazer mais agilidade e menores custos para os exportadores e importadores do país, além de ampliar a competitividade da indústria nacional.

\section{Referências}

ANDERSON, J. A Theoretical Foundation for the Gravity Equation. American Economic Review, American Economic Association, Nashville, v. 69, n. 1, 1979. Disponível em: http://ideas.repec.org/a/aea/aecrev/v69y1979ilp106-16.html. Acesso em: 20 maio 2008.

ANDERSON, J.; WINCOOP E. Gravity with Gravitas: A Solution to the Border Puzzle. American Economic Review, American Economic Association, Nashville, v. 93, n. 1, 2003. Disponível em: http://ideas.repec.org/p/boc/bocoec/485. html. Acesso em: 20 maio 2008.

. Trade Costs. Boston, 2004. (Boston College Working Papers in Economics 593). Disponível em: http://ideas.repec.org/p/boc/bocoec/593.html. Acesso em: 20 maio 2008.

ANDERSON, J.; YOTOV, Y. The Changing Incidence of Geography. Cambridge, 2008. (NBER Working Paper No. W14423). Disponível em: http://ssrn.com/ abstract $=1288422$. Acesso em: 28 novembro 2008.

APEC. Assessing APEC Trade Liberalization and Facilitation - 1999 Update. Cingapura, 1999. (APEC Economic Committee). Disponível em: http://www.apec. org/apec/publications. Acesso em: 05 maio 2008. 
. Trade Facilitation and Trade Liberalization: From Shanghai to Bogor. Cingapura, 2004. (APEC Economic Committee). Disponível em: http://www.apec. org/apec/publications. Acesso em: 05 maio 2008.

BALDWIN, R.E.; TAGLIONI, D. Gravity for Dummies and Dummies for Gravity Equations. Londres, 2006. (CEPR Discussion Paper No. 5850). Disponível em: http://ssrn.com/abstract=945443. Acesso em: 12 maio 2008.

BANCO MUNDIAL. World Development Indicators Online (WDI). Disponível em: http://www.worldbank.org/ . Acesso em: 13 junho 2008.

BEHRENS, K., ERTUR, C.; KOCH, W. 'Dual' Gravity: Using Spatial Econometrics to Control for Multilateral Resistance. Belgica, 2007. (CORE Discussion Paper No. 2007/59). Disponível em: http://ssrn.com/abstract=101. Acesso em: 28 novembro 2008.

BERGSTRAND, J. H. The Generalized Gravity Equation, Monopolistic Competition, and the Factor-Proportions Theory in International Trade. The Review of Economics and Statistics, Cambridge, v. 71, n. 1, pages 143-53, Fevereiro, 1989.

CEPII. Centre D'Estudes Prospectives et d'Informations Internationales. Disponível em: http://www.cepii.fr/anglaisgraph/news/accueilengl.htm. Acesso em: 28 novembro 2008.

CHENG, I.; WALL, H. J. Controlling for Heterogeneity in Gravity Models of Trade and Integration. Federal Reserve Bank of St. Louis Review, St. Louis, v. 87, n. 1, 2005. Disponível em: http://ssrn.com/abstract=656201. Acesso em: 28 novembro 2008.

COMTRADE. United Nations Commodity Trade Statistics Database. Disponível em: http://comtrade.un.org/db. Acesso em: 13 junho 2008.

DEARDORFF, A.V. Determinants of Bilateral Trade: Does Gravity Work in a Neoclassical World? Cambridge, 1995. (NBER Working Paper No. W5377). Disponível em: http://ssrn.com/abstract=93748. Acesso em: 20 maio 2008.

DISDIER, A.; FONTAGNÉ, L.; MIMOUNI, M. The Impact of Regulations on Agricultural Trade: Evidence from the SPS and TBT Agreements. American Journal of Agricultural Economics, Oxford, v. 90, n. 2, 2008. Disponível em: http://ssrn.com/abstract=1119018. Acesso em: 17 abril 2008.

DOING BUSINESS. Doing Business in 2007: How to Reform. World Bank, Washington, 2007. Disponível em: www.doingbusiness.org. Acesso em: 13 junho 2008.

DUVAL, Y. Cost and Benefits of Implementing Trade Facilitation Measures under Negotiations at the WTO: an Exploratory Survey. Bangkok, 2006. Working Papers 306 from Asia-Pacific Research and Training Network on Trade - ARTNeT. Disponível em: http://ideas.repec.org/p/esc/wpaper/306.html. Acesso em: 23 janeiro 2008. 
FEENSTRA, R. C. Advanced International Trade: Theory and Evidence. Princeton: Princeton University Press, 2004, 484 p.

FOX, A.K.; T.W.; FRANCOIS, J.; LONDONO-KENT, P. Measuring Border Crossing costs and their Impact on Trade Flows: The United States-Mexican Trucking Case. West Lafayette, 2003. (GTAP Resource \# 1282). Disponível em: https://www.gtap.agecon.purdue.edu/resources/res_display.asp?RecordID =1282. Acesso em: 22 abril 2008.

FRANCOIS J.; MEIJL, H. van; TONGEREN, F. van. Trade Liberalization in the Doha Development Round. Economic Policy, Londres, v. 20, n. 42, 2005. Disponível em: http://ideas.repec.org/a/bla/ecpoli/v20y2005i42p349-391.html. Acesso em: 22 abril 2008

GREENE, W. H. Econometric Analysis. 2. ed. New York: Macmillan, 1993. 791p.

HELBLE, M.; SHEPHERD, B.; WILSON, J.S. Transparency and Trade Facilitation in the Asia Pacific: Estimating the Gains from Reform. Washington D.C.: World Bank Development Research Group, 2007. Disponível em: http http://www. worldbank.org. Acesso em: 23 janeiro 2008.

HERTEL, T.W.; WALMSLEY, T.; ITAKURA, K. 2001. Dynamic Effects of the "New Age" Free Trade Agreement between Japan and Singapore. West Lafayette, 2001. (GTAP Working Papers 823). Disponível em: http://ideas.repec.org/p/ gta/workpp/823.html. Acesso em: 20 abril 2008.

KEE, H.L.; NICITA, A.; OLARREAGA, M. Estimating Trade Restrictiveness Indices. Washington D.C., 2006. (World Bank Policy Research Working Paper No. 3840). Disponível em: http://ideas.repec.org/p/wbk/wbrwps/3840.html. Acesso em: 15 maio 2008.

LIU, X. GATT/WTO Promotes Trade Strongly: Sample Selection and Model Specification. In: 10th Annual Conference on Global Economic Analysis, West Lafayette, 2007. 10th Annual Conference on Global Economic Analysis. West Lafayette: Purdue University, 2007.

LOGISTICS PERFORMANCE INDEX. Connecting to Compete: Trade Logistics in the Global Economy, The Logistics Performance Index and Its Indicators. World Bank, Washington, 2007. Disponível em: http http://www.worldbank. org. Acesso em: 13 junho 2008.

MACMAP. Market Access Map - UNCTAD/WTO. Disponível em: http://www. macmap.org. Acesso em: 13 junho 2008.

MARTINEZ-ZARZOSO, I.; NOWAK-LEHMANN, F.; VOLLMER, S. The Log of Gravity Revisited. Goettingen, 2006. (University of Goettingen Working Paper). Disponível em: http://ssrn.com/abstract=999908. Acesso em: 28 novembro 2008.

OECD. Quantitative Assessment of the Benefits of Trade Facilitation. Paris, 2003. (OECD Trade Policy Working Papers TD/TD/WP(2003)31/FINAL). Disponível em: http://ideas.repec.org/p/wpa/wuwpit/0401008.html. Acesso em: 20 abril 2008. 
. The Economic Impact of Trade Facilitation. Paris, 2005. (OECD Trade Policy Working Papers Working Paper TD/TC/WP(2005)12/FINAL). Disponível em: http://ideas.repec.org/p/oec/traaab/21-en.html. Acesso em: 19 abril 2008.

OMC. Disponível em: http://www.wto.org/english/tratop_e/tradfa_e/tradfa_e.htm. Acesso em: 14 fevereiro 2008.

SADIKOV, A.M. Border and Behind-the-Border Trade Barriers and Country Exports. Washington D. C., 2007. (IMF Working Paper No. 07/292). Disponível em: http://ssrn.com/abstract =1079220. Acesso em: 11 fevereiro 2008.

SANTOS SILVA, J. M. C.; TENREYRO S. The Log of Gravity. The Review of Economics and Statistics, Cambridge, MIT Press, vol. 88, n. 4, 2006. Disponível em: http://ideas.repec.org/a/tpr/restat/v88y2006i4p64l-658.html. Acesso em: 28 novembro 2008.

SHEPHERD, B.; WILSON, J.S. Trade Facilitation in ASEAN Member Countries: measuring progress and assessing priorities. Washington D.C., 2008. (World Bank Policy Research Working Paper No. 4615). Disponível em: http http:// www.worldbank.org. Acesso em: 18 junho 2008.

SILIVERSTOVS, B.; SCHUMACHER D. Estimating Gravity Equations : To Log or Not to Log? Berlin, 2007. (Discussion Papers of DIW Berlin 739). Disponível em: http://ideas.repec.org/p/diw/diwwpp/dp739.html. Acesso em: 28 novembro 2008.

TINBERGEN, J. Shaping the World Economy: Suggestions for an International Economic Policy. Nova York: Twentieth Century Fund, 1962, 330 p.

UNCTAD. Electronic Commerce and Developing Countries: A Computable General Equilibrium Analysis. In: E-commerce and Development Report 2001. Genebra, 2001. Disponível em: http://r0.unctad.org/ecommerce/docs/edr01_en.htm. Acesso em: 05 maio 2008.

WILSON, J.S., MANN, C.L.; OTSUKI, T. Trade Facilitation and Economic Development: Measuring the Impact. Washington D.C., 2003. (World Bank Policy Research Working Paper No. 2988). Disponível em: http://ssrn.com/ abstract $=636350$. Acesso em: 12 março 2008.

. Assessing the Potential Benefit of Trade Facilitation: A Global Perspective. Washington D.C., 2004. (World Bank Policy Research Working Paper No. 3224). Disponível em: http://ssrn.com/abstract=610266. Acesso em: 12 março 2008. 


\section{ANEXO 1 - Relação dos Países Considerados para Análise, Apresentados com Respectivos Códigos das Nações Unidas e da ISO}

\begin{tabular}{|c|c|c|}
\hline PAÍS & CÓDIGO UN & CÓDIGO ISO \\
\hline Argentina & 32 & ARG \\
\hline Austrália & 36 & AUS \\
\hline Áustria & 40 & AUT \\
\hline Bélgica & 56 & BEL \\
\hline Brasil & 76 & BRA \\
\hline Bulgária & 100 & BGR \\
\hline Canadá & 124 & CAN \\
\hline Chile & 152 & $\mathrm{CHL}$ \\
\hline China & 156 & $\mathrm{CHN}$ \\
\hline Chipre & 196 & CYP \\
\hline República Checa & 203 & CZE \\
\hline Dinamarca & 208 & DNK \\
\hline Estônia & 233 & EST \\
\hline Finlândia & 246 & FIN \\
\hline França & 251 & FRA \\
\hline Alemanha & 276 & DEU \\
\hline Grécia & 300 & GRC \\
\hline Hungria & 348 & HUN \\
\hline Irlanda & 372 & IRL \\
\hline Itália & 381 & ITA \\
\hline Japão & 392 & JPN \\
\hline Letônia & 428 & LVA \\
\hline Lituânia & 440 & LTU \\
\hline Luxemburgo & 442 & LUX \\
\hline Malta & 470 & MLT \\
\hline México & 484 & MEX \\
\hline Holanda & 528 & NLD \\
\hline Nova Zelândia & 554 & NZL \\
\hline Paraguai & 600 & PRY \\
\hline Polônia & 616 & POL \\
\hline Portugal & 620 & PRT \\
\hline Rep. da Coréia & 410 & KOR \\
\hline Romênia & 642 & ROM \\
\hline Rússia & 643 & RUS \\
\hline Arábia Saudita & 682 & SAU \\
\hline República Eslovaca & 703 & SVK \\
\hline Eslovênia & 705 & SVN \\
\hline África do Sul & 710 & ZAF \\
\hline Espanha & 724 & ESP \\
\hline Suécia & 752 & SWE \\
\hline Reino Unido & 826 & GBR \\
\hline Estados Unidos & 842 & USA \\
\hline Uruguai & 858 & URY \\
\hline
\end{tabular}

Fonte: MacMap. 